\title{
Water Trapping Dynamics in Carbohydrate-Populated Smectite Interlayer Nanopores
}

Sabrina E. Kelch ${ }^{a}$, Eric Ferrage ${ }^{b}$, Bruno Lenson, Laurent Charlet ${ }^{c}$, Ludmilla Aristilde La,de,* $^{a}$

a Soil and Crop Sciences Section, School of Integrative Plant Science, College of Agriculture and Life Sciences, Cornell University, Ithaca, NY 14853, USA

${ }^{\mathrm{b}}$ Institut de Chimie des Milieux et Matériaux de Poitiers (IC2MP), CNRS-Université de Poitiers, Poitiers, France c Univ. Grenoble Alpes, Univ. Savoie Mont Blanc, CNRS, IRD, IFSTTAR, ISTerre, F-38000 Grenoble, France

d Department of Biological and Environmental Engineering, College of Agriculture and Life Sciences, Cornell University, Ithaca, NY 14853, USA

e Department of Civil and Environmental Engineering, McCormick School of Engineering and Applied Science, Northwestern University, Evanston, II 60208, USA

*Corresponding Author:ludmilla.aristilde@northwestern.edu; Phone: 847-491-2999 
Table S1. Results from adsorption experiments and X-ray diffraction patterns of montmorillonite (MONT) reacted with cellobiose a.

\begin{tabular}{|c|c|c|c|c|c|c|c|c|c|c|c|c|c|c|c|c|c|c|c|c|c|}
\hline \multirow{2}{*}{$\begin{array}{c}\text { amount adsorbed } \\
\text { \%RH }\end{array}$} & \multicolumn{3}{|c|}{$0 \mu \mathrm{mol} g-1$ clay } & \multicolumn{3}{|c|}{$0.66 \mu \mathrm{mol} g-1$ clay } & \multicolumn{3}{|c|}{$2.19 \mu \mathrm{mol} g-1$ clay } & \multicolumn{3}{|c|}{$2.64 \mu \mathrm{mol} g-1$ clay } & \multicolumn{3}{|c|}{$4.14 \mu \mathrm{mol} g-1$ clay } & \multicolumn{3}{|c|}{$18.33 \mu \mathrm{mol} g-1$ clay } & \multicolumn{3}{|c|}{$33.23 \mu \mathrm{mol} g-1$ clay } \\
\hline & $d_{001}$ & $\Delta \mathrm{d}$ & fwhm & $d_{001}$ & $\Delta \mathrm{d}$ & fwhm & $d_{001}$ & $\Delta \mathrm{d}$ & fwhm & $d_{001}$ & $\Delta \mathrm{d}$ & fwhm & $d_{001}$ & $\Delta \mathrm{d}$ & fwhm & $d_{001}$ & $\Delta \mathrm{d}$ & fwhm & $d_{001}$ & $\Delta \mathrm{d}$ & fwhm \\
\hline 95 & 1.55 & - & 0.39 & 1.55 & - & 0.39 & 1.55 & - & 0.45 & 1.54 & & 0.40 & 1.55 & - & 0.39 & 1.55 & - & 0.43 & 1.59 & & 0.92 \\
\hline 90 & 1.55 & 0.007 & 0.39 & 1.55 & 0.006 & 0.38 & 1.55 & 0.003 & 0.44 & 1.54 & 0.001 & 0.40 & 1.54 & 0.006 & 0.38 & 1.54 & 0.009 & 0.44 & 1.57 & 0.022 & 0.39 \\
\hline 80 & 1.54 & 0.015 & 0.39 & 1.53 & 0.022 & 0.38 & 1.54 & 0.012 & 0.44 & 1.53 & 0.013 & 0.40 & 1.54 & 0.012 & 0.39 & 1.52 & 0.025 & 0.47 & 1.52 & 0.065 & 0.39 \\
\hline 70 & 1.50 & 0.057 & 0.71 & 1.54 & 0.013 & 0.43 & 1.49 & 0.064 & 0.89 & 1.50 & 0.04 & 0.73 & 1.49 & 0.063 & 0.77 & 1.49 & 0.055 & 0.79 & 1.52 & 0.071 & 0.39 \\
\hline 60 & 1.26 & 0.296 & 0.77 & 1.49 & 0.058 & 0.77 & 1.26 & 0.293 & 0.77 & 1.25 & 0.288 & 0.66 & 1.26 & 0.29 & 0.67 & 1.29 & 0.258 & 1.3 & 1.49 & 0.096 & 0.71 \\
\hline 50 & 1.25 & 0.302 & 0.43 & 1.25 & 0.297 & 0.45 & 1.26 & 0.297 & 0.46 & 1.25 & 0.296 & 0.44 & 1.25 & 0.296 & 0.43 & 1.27 & 0.281 & 0.66 & 1.28 & 0.304 & 0.77 \\
\hline 40 & 1.24 & 0.315 & 0.59 & 1.24 & 0.313 & 0.46 & 1.25 & 0.308 & 0.53 & 1.24 & 0.302 & 0.45 & 1.24 & 0.306 & 0.47 & 1.27 & 0.283 & 0.61 & 1.27 & 0.315 & 0.62 \\
\hline 30 & 1.17 & 0.382 & 0.92 & 1.22 & 0.335 & 0.74 & 1.22 & 0.332 & 0.81 & 1.22 & 0.324 & 0.70 & 1.21 & 0.337 & 0.78 & 1.26 & 0.291 & 0.79 & 1.27 & 0.321 & 0.79 \\
\hline 20 & 1.12 & 0.429 & 0.79 & 1.13 & 0.422 & 0.81 & 1.14 & 0.419 & 0.99 & 1.14 & 0.404 & 0.87 & 1.13 & 0.423 & 0.85 & 1.21 & 0.339 & 1.4 & 1.25 & 0.338 & 1.4 \\
\hline
\end{tabular}

a Equilibrated Relative Humidity (\%RH) the percent humidity the sample was equilibrated at in the X-ray diffractometer, Full Width Half-

Maximum (fwhm) the width of the $d_{001}$ peak at half height shown in $2 \theta$ degrees, $d_{001}$ the basal spacing of the clay layers shown in nm units

Table S2. Results from adsorption experiments and X-ray diffraction patterns of montmorillonite (MONT) reacted with glucose ${ }^{b}$.

\begin{tabular}{|c|c|c|c|c|c|c|c|c|c|c|c|c|c|c|c|c|c|c|c|c|c|}
\hline \multirow{2}{*}{$\begin{array}{c}\text { amount adsorbed } \\
\text { \%RH }\end{array}$} & \multicolumn{3}{|c|}{$0 \mu \mathrm{mol} g-1$ clay } & \multicolumn{3}{|c|}{$2.08 \mu \mathrm{mol} g-1$ clay } & \multicolumn{3}{|c|}{$3.13 \mu \mathrm{mol} g-1$ clay } & \multicolumn{3}{|c|}{$3.42 \mu \mathrm{mol}$ g-1 clay } & \multicolumn{3}{|c|}{$3.47 \mu \mathrm{mol} g-1$ clay } & \multicolumn{3}{|c|}{$16.64 \mu \mathrm{mol} g-1$ clay } & \multicolumn{3}{|c|}{$30.86 \mu \mathrm{mol}$ g-1 clay } \\
\hline & $d_{001}$ & $\Delta \mathrm{d}$ & fwhm & $d_{001}$ & $\Delta \mathrm{d}$ & fwhm & $d_{001}$ & $\Delta d$ & fwhm & $d_{001}$ & $\Delta d$ & fwhm & $d_{001}$ & $\Delta d$ & fwhm & $d_{001}$ & $\Delta d$ & fwhm & $d_{001}$ & $\Delta \mathrm{d}$ & fwhm \\
\hline 95 & 1.55 & - & 0.39 & 1.55 & - & 0.41 & 1.56 & - & 0.41 & 1.56 & - & 0.42 & 1.55 & - & 0.40 & 1.55 & - & 0.41 & 1.55 & - & 0.41 \\
\hline 90 & 1.55 & 0.007 & 0.39 & 1.55 & 0.004 & 0.40 & 1.55 & 0.013 & 0.40 & 1.56 & 0 & 0.41 & 1.55 & 0.005 & 0.40 & 1.54 & 0.003 & 0.41 & 1.54 & 0.005 & 0.40 \\
\hline 80 & 1.54 & 0.015 & 0.39 & 1.54 & 0.017 & 0.40 & 1.53 & 0.03 & 0.40 & 1.53 & 0.028 & 0.40 & 1.55 & 0.005 & 0.40 & 1.53 & 0.017 & 0.39 & 1.54 & 0.009 & 0.41 \\
\hline 70 & 1.50 & 0.057 & 0.71 & 1.50 & 0.05 & 0.68 & 1.45 & 0.115 & 1.2 & 1.50 & 0.058 & 0.75 & 1.51 & 0.041 & 0.64 & 1.49 & 0.057 & 0.64 & 1.49 & 0.054 & 0.63 \\
\hline 60 & 1.26 & 0.296 & 0.77 & 1.27 & 0.285 & 1.4 & 1.26 & 0.296 & 1.0 & 1.26 & 0.298 & 1.2 & 1.24 & 0.308 & 0.80 & 1.26 & 0.287 & 1.5 & 1.41 & 0.138 & 1.4 \\
\hline 50 & 1.25 & 0.302 & 0.43 & 1.25 & 0.305 & 0.46 & 1.25 & 0.315 & 0.58 & 1.25 & 0.312 & 0.46 & 1.24 & 0.315 & 0.58 & 1.26 & 0.291 & 0.62 & 1.28 & 0.264 & 1.2 \\
\hline 40 & 1.24 & 0.315 & 0.59 & 1.25 & 0.309 & 0.46 & 1.24 & 0.324 & 0.61 & 1.25 & 0.312 & 0.45 & 1.23 & 0.319 & 0.60 & 1.25 & 0.302 & 0.57 & 1.27 & 0.278 & 1.0 \\
\hline 30 & 1.17 & 0.382 & 0.92 & 1.24 & 0.317 & 0.67 & 1.22 & 0.341 & 0.71 & 1.23 & 0.332 & 0.68 & 1.22 & 0.331 & 0.64 & 1.23 & 0.318 & 0.85 & 1.27 & 0.281 & 1.3 \\
\hline 20 & 1.12 & 0.429 & 0.79 & 1.16 & 0.392 & 1.0 & 1.15 & 0.41 & 0.88 & 1.17 & 0.392 & 0.97 & 1.16 & 0.394 & 0.94 & 1.16 & 0.391 & 1.2 & 1.19 & 0.36 & 1.8 \\
\hline
\end{tabular}

${ }^{\mathrm{b}}$ Equilibrated Relative Humidity (\%RH) the percent humidity the sample was equilibrated at in the X-ray diffractometer, Full Width Half-

Maximum ( $\mathrm{fwhm}$ ) the width of the $d_{001}$ peak at half height shown in $2 \theta$ degrees, $d_{001}$ the basal spacing of the clay layers shown in nm units 


\section{Supplementary Information - Part B}
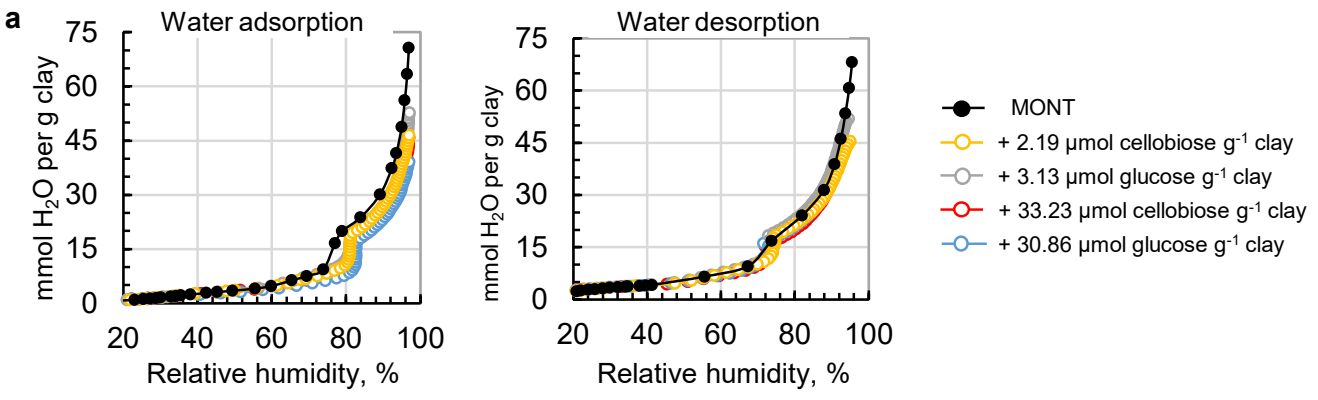

b

-o- Water adsorption - - Water desorption

$$
2.19
$$

33.23

3.13

30.86
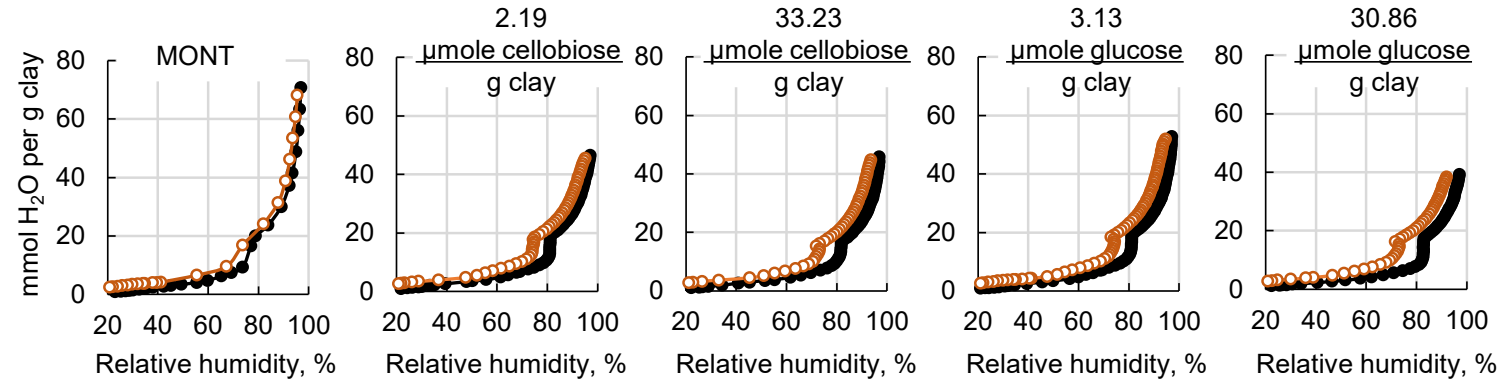

Relative humidity, \%

Relative humidity, \%

Relative humidity, \%

Relative humidity, \%

Relative humidity, \%

Figure S1. Carbohydrate-influenced water adsorption and desorption in montmorillonite (MONT). (a) Isotherms of water adsorption (left) and desorption (right) as a function of relative humidity obtained for montmorillonite alone or with different carbohydrate loadings. (b) comparison of water adsorption versus desorption profiles at each carbohydrate loading. All graphs are corrected for carbohydrate content (per gram dry clay with carbohydrate subtracted). 


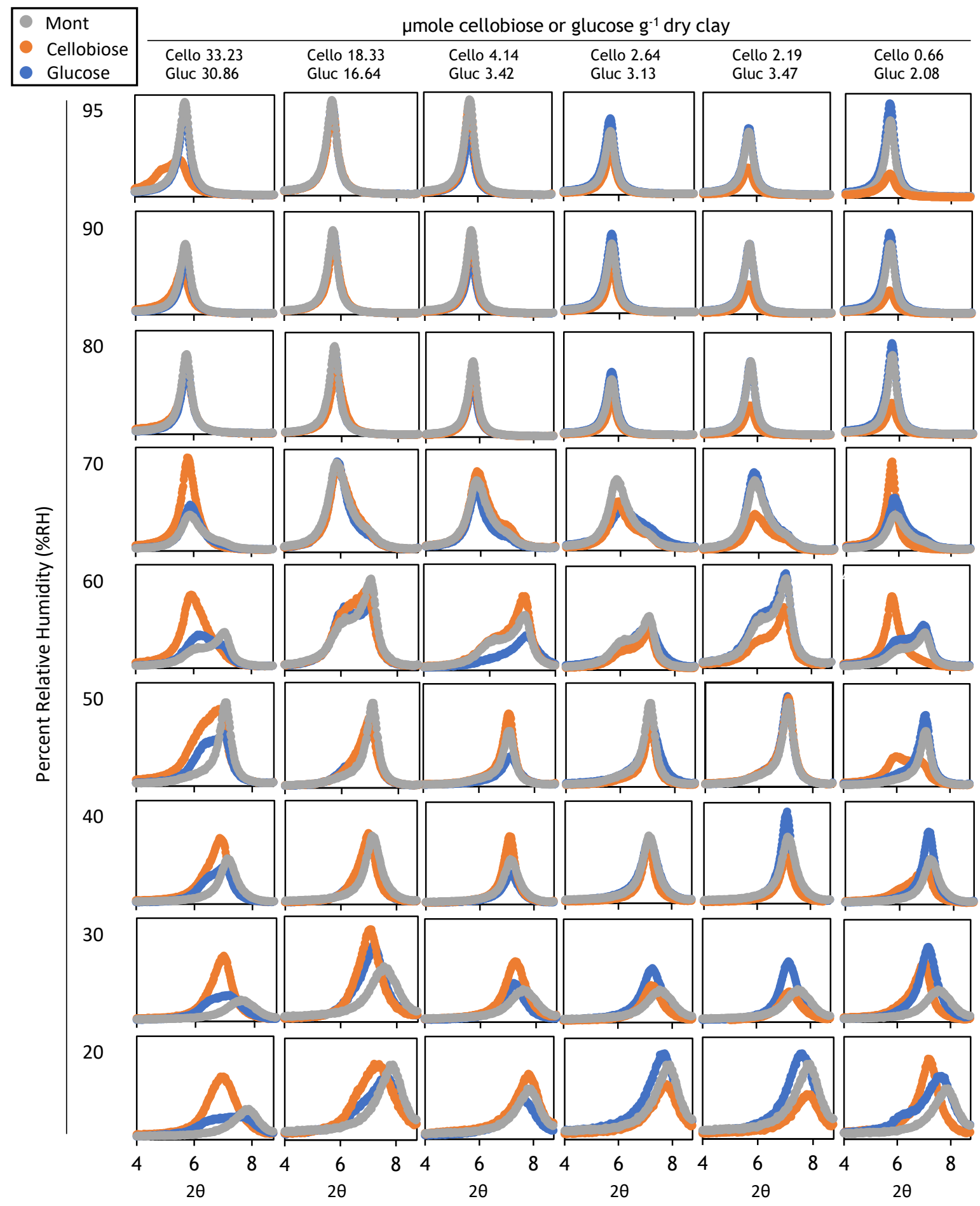

Figure S2. Moisture-dependent X-ray diffraction profiles. X-ray diffraction patterns of montmorillonite alone or loaded with cellobiose or glucose (adsorbed amounts are shown $\mu$ mol carbohydrate per gram of dry clay). Samples were conditioned at different relative humidity $(\mathrm{RH})$, from top to bottom: $95 \%, 90 \%, 80 \%, 70 \%$, $60 \%, 50 \%, 40 \%, 30 \%$, and $20 \%$. 


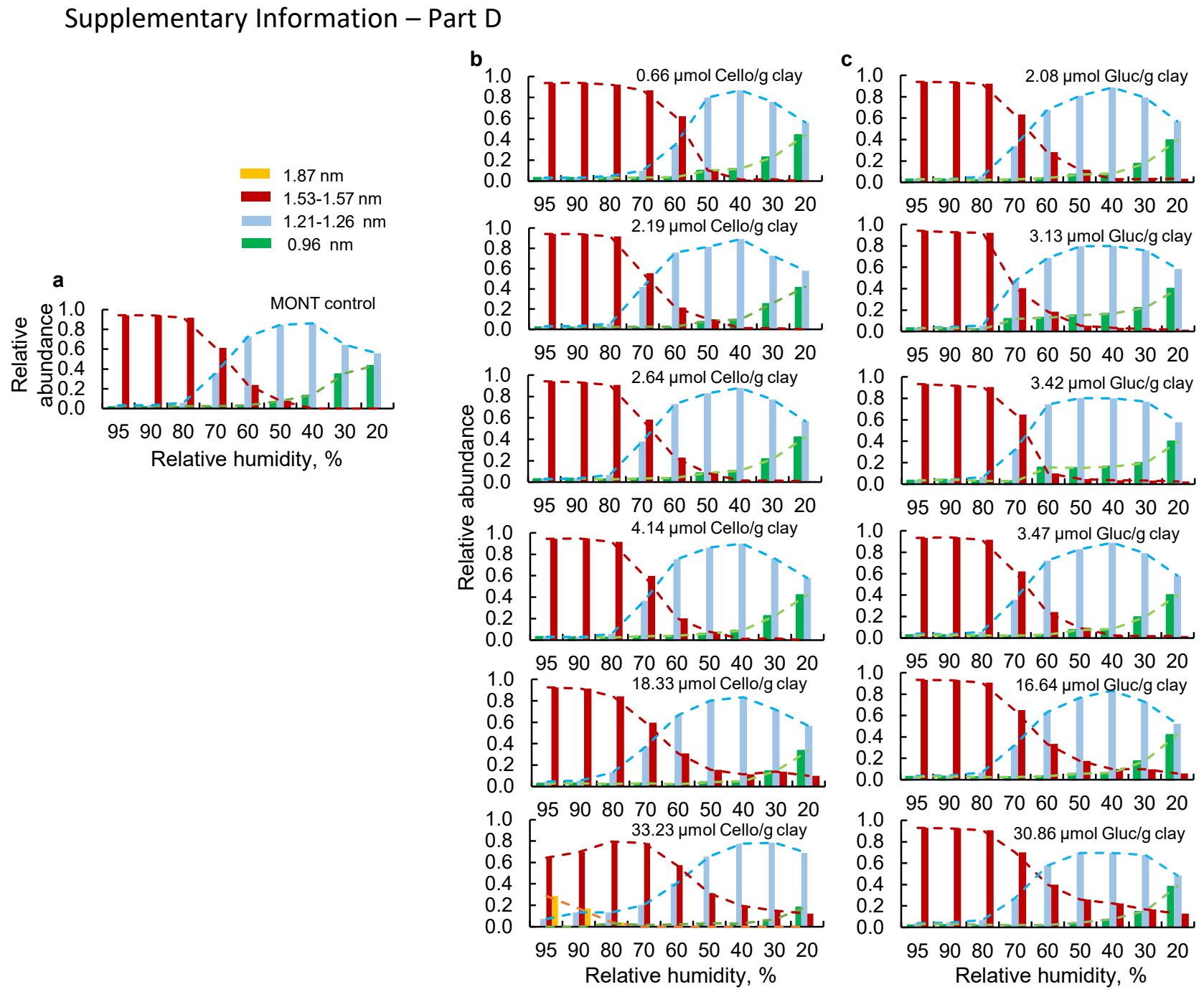

Figure S3. Modeling moisture-dependent X-ray diffraction profile of nanopore distribution. Relative nanopore abundance of (a) montmorillonite alone or loaded with (b) cellobiose or (c) glucose. In b and c, loadings are shown in $\mu \mathrm{mol}$ carbohydrate per gram of dry clay. The different interlayer sizes used in the modeling are represented by green bars $(0.96 \mathrm{~nm})$, light blue bars $(1.21-1.26 \mathrm{~nm})$, dark red bars (1.53-1.57 $\mathrm{nm})$, and yellow bars (1.87 $\mathrm{nm})$. 


\section{Supplementary Information - Part E}

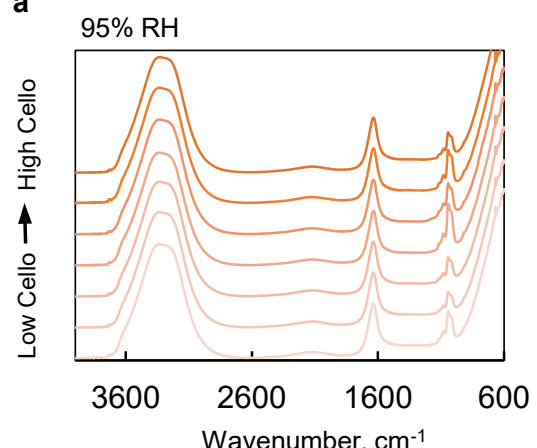

b

$75 \% \mathrm{RH}$

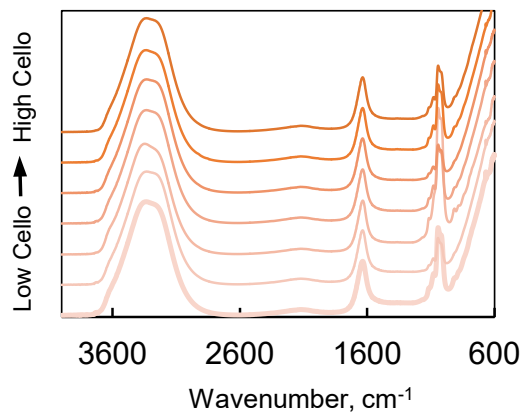

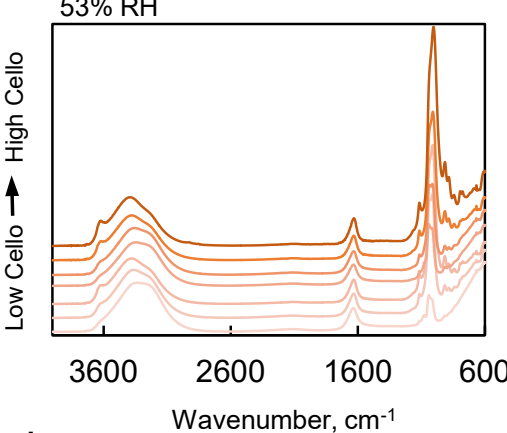

$33 \% \mathrm{RH}$

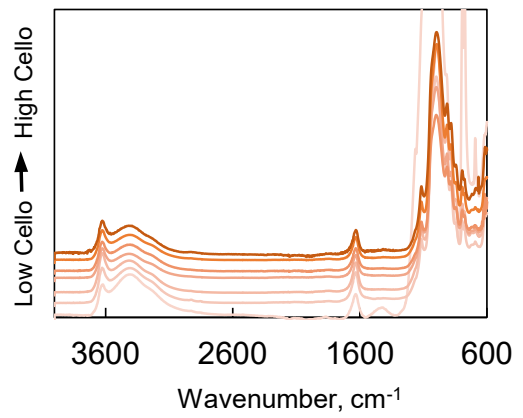

e

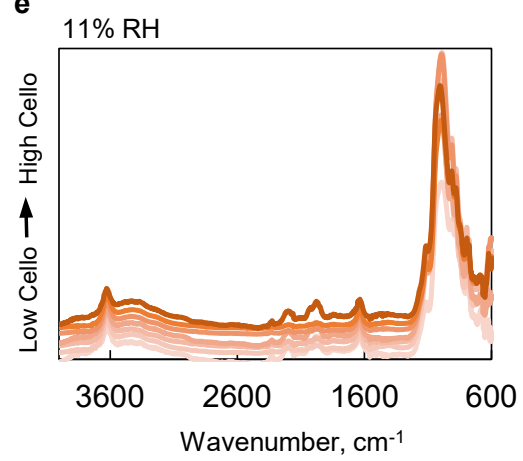

Figure S4. Loss of exchangeable water during dehydration in cellobiose-smectite nanoaggregates. Attenuated total reflectance Fourier-transform infrared (ATR-FTIR) spectra of montmorillonite (MONT) in the presence of increasing carbohydrate concentrations of cellobiose equilibrated at different $\mathrm{RH}: 95 \%, 75 \%, 53 \%, 33 \%, 11 \%$. From bottom to top in each panel, MONT loaded with cellobiose ( $\mu$ mol cellobiose/gram dry clay) 0, 2.19, 2.64, 4.14, 18.33, and $33.23 \mu \mathrm{mol}$ per gram. All spectra normalized to the maximum intensity. 


\section{Supplementary Information - Part F}

a
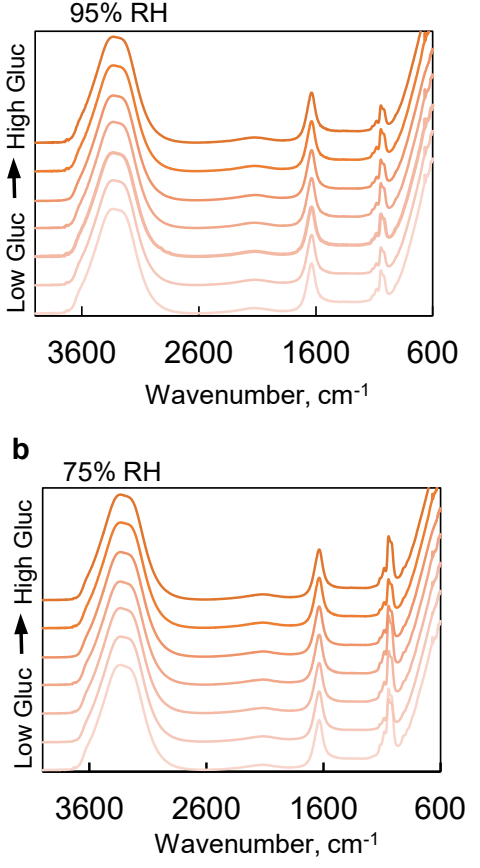

C

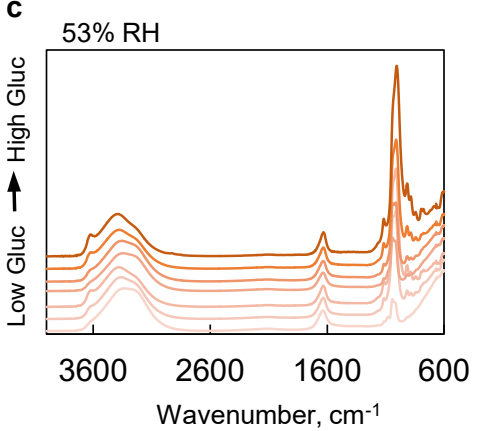

d

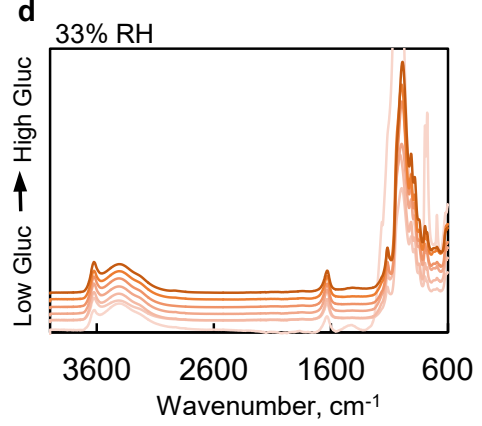

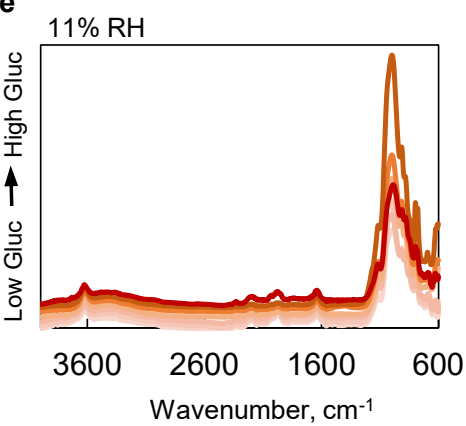

Figure S5. Loss of exchangeable water during dehydration in cellobiose-smectite nanoaggregates. Attenuated total reflectance Fourier-transform infrared (ATR-FTIR) spectra of montmorillonite (MONT) in the presence of increasing carbohydrate concentrations of glucose equilibrated at different $\mathrm{RH}: 95 \%, 75 \%, 53 \%, 33 \%, 11 \%$. From bottom to top in each panel, MONT loaded with glucose ( $\mu \mathrm{mol}$ glucose/gram dry clay) $0,3.13,3.42,3.47,16.64$, and $30.86 \mu \mathrm{mol}$ per gram. All spectra normalized to the maximum intensity. 


\section{Supplementary Information - Part G}

Table S3. Results from Fourier-transform infrared experiments of montmorillonite (MONT) reacted with cellobiose a.

\begin{tabular}{|c|c|c|c|c|c|c|c|c|c|c|c|c|c|c|c|c|c|c|c|c|c|c|c|c|c|c|c|c|c|c|c|c|c|}
\hline \multirow[b]{2}{*}{ amount adsorbed } & \multicolumn{3}{|c|}{$\underline{0 \text { minutes }}$} & \multicolumn{3}{|c|}{10 minutes } & \multicolumn{3}{|c|}{20 minutes } & \multicolumn{3}{|c|}{30 minutes } & \multicolumn{3}{|c|}{40 minutes } & \multicolumn{3}{|c|}{$\underline{50 \text { minutes }}$} & \multicolumn{3}{|c|}{60 minutes } & \multicolumn{3}{|c|}{70 minutes } & \multicolumn{3}{|c|}{80 minutes } & \multicolumn{3}{|c|}{90 minutes } & \multicolumn{3}{|c|}{100 minutes } \\
\hline & $\begin{array}{c}\text { mineral } \\
\mathrm{OH}\end{array}$ & $\begin{array}{c}\text { free } \\
\mathrm{OH}\end{array}$ & ratio & $\underset{\mathrm{OH}}{\text { mineral }}$ & $\begin{array}{l}\text { free } \\
\mathrm{OH}\end{array}$ & ratio & $\begin{array}{c}\text { mineral } \\
\mathrm{OH}\end{array}$ & $\begin{array}{c}\text { free } \\
\mathrm{OH}\end{array}$ & ratio & $\begin{array}{c}\text { mineral } \\
\mathrm{OH}\end{array}$ & $\begin{array}{l}\text { free } \\
\mathrm{OH}\end{array}$ & ratio & $\begin{array}{c}\text { mineral } \\
\mathrm{OH}\end{array}$ & $\begin{array}{c}\text { free } \\
\mathrm{OH}\end{array}$ & ratio & $\begin{array}{c}\text { mineral } \\
\mathrm{OH}\end{array}$ & $\begin{array}{c}\text { free } \\
\mathrm{OH}\end{array}$ & ratio & $\begin{array}{c}\text { mineral } \\
\mathrm{OH}\end{array}$ & $\begin{array}{c}\text { free } \\
\mathrm{OH}\end{array}$ & ratio & $\begin{array}{c}\text { mineral } \\
\mathrm{OH}\end{array}$ & $\begin{array}{c}\text { free } \\
\mathrm{OH}\end{array}$ & ratio & $\begin{array}{c}\text { mineral } \\
\mathrm{OH}\end{array}$ & $\begin{array}{c}\text { free } \\
\mathrm{OH}\end{array}$ & ratio & $\begin{array}{c}\text { mineral } \\
\mathrm{OH}\end{array}$ & $\begin{array}{c}\text { free } \\
\mathrm{OH}\end{array}$ & ratio & $\underset{\mathrm{OH}}{\operatorname{mineral}}$ & $\begin{array}{c}\text { free } \\
\mathrm{OH}\end{array}$ & ratio \\
\hline 0 pmole $\mathrm{g}^{-1}$ & 0.00 & 0.27 & 0.00 & 0.00 & 0.27 & 0.00 & 0.00 & 0.27 & 0.00 & 0.00 & 0.27 & 0.00 & 0.00 & 0.27 & 0.00 & 0.00 & 0.23 & 0.00 & 0.04 & 0.13 & 3.00 & 0.03 & 0.07 & 2.57 & 0.03 & 0.03 & 1.00 & 0.03 & 0.02 & 0.70 & 0.03 & 0.02 & 0.56 \\
\hline 0.66 pmole $\mathrm{g}^{-1}$ & 0.00 & 0.26 & 0.00 & 0.00 & 0.26 & 0.00 & 0.00 & 0.25 & 0.00 & 0.00 & 0.25 & 0.00 & 0.08 & 0.24 & 3.00 & 0.09 & 0.20 & 2.22 & 0.13 & 0.14 & 1.08 & 0.13 & 0.12 & 0.92 & 0.11 & 0.07 & 0.64 & 0.11 & 0.07 & 0.64 & 0.10 & 0.07 & 0.70 \\
\hline $2.19 \mu^{2}$ ole $\mathrm{g}^{-1}$ & 0.00 & 0.26 & 0.00 & 0.00 & 0.26 & 0.00 & 0.00 & 0.25 & 0.00 & 0.00 & 0.24 & 0.00 & 0.08 & 0.22 & 2.74 & 0.09 & 0.20 & 2.22 & 0.13 & 0.13 & 1.02 & 0.12 & 0.09 & 0.78 & 0.11 & 0.07 & 0.59 & 0.11 & 0.06 & 0.56 & 0.11 & 0.06 & 0.55 \\
\hline $2.64 \mu^{2}$ mole $\mathrm{g}^{-1}$ & 0.00 & 0.26 & 0.00 & 0.00 & 0.26 & 0.00 & 0.00 & 0.25 & 0.00 & 0.00 & 0.25 & 0.00 & 0.08 & 0.24 & 3.11 & 0.09 & 0.20 & 2.22 & 0.13 & 0.14 & 1.08 & 0.13 & 0.09 & 0.74 & 0.11 & 0.07 & 0.59 & 0.11 & 0.06 & 0.55 & 0.11 & 0.06 & 0.55 \\
\hline 4.14 нmole $\mathrm{g}^{-1}$ & 0.00 & 0.27 & 0.00 & 0.00 & 0.27 & 0.00 & 0.00 & 0.27 & 0.00 & 0.00 & 0.26 & 0.00 & 0.00 & 0.25 & 0.00 & 0.07 & 0.22 & 3.19 & 0.07 & 0.19 & 2.71 & 0.08 & 0.18 & 2.21 & 0.08 & 0.17 & 2.07 & 0.09 & 0.15 & 1.71 & 0.10 & 0.06 & 0.59 \\
\hline $18.32 \mu$ mole $\mathrm{g}^{-1}$ & 0.00 & 0.26 & 0.00 & 0.00 & 0.26 & 0.00 & 0.00 & 0.25 & 0.00 & 0.07 & 0.22 & 3.14 & 0.10 & 0.20 & 2.00 & 0.10 & 0.18 & 1.83 & 0.10 & 0.16 & 1.60 & 0.11 & 0.13 & 1.14 & 0.10 & 0.07 & 0.66 & 0.09 & 0.05 & 0.58 & 0.09 & 0.05 & 0.57 \\
\hline $33.23 \mu$ mole g & 0.00 & 0.26 & 0.00 & 00 & 0.26 & 0.00 & 0.00 & 0.25 & 0.00 & 0.00 & 0.23 & 0.00 & .08 & 0.18 & 2.40 & 0.12 & 0.12 & 0.98 & 0.06 & 0.04 & 0.60 & 0.05 & 0.03 & 0.56 & 0.05 & 0.03 & 0.56 & 0.05 & 0.03 & 0.56 & 0.05 & 0.03 & D.56 \\
\hline
\end{tabular}

${ }^{a} y$-axis value (or absorbance) of the attenuated total reflectance Fourier-transform infrared (ATR-FTIR) spectra vibrational bands of interest: the mineral -OH vibrational band at $\sim 3615 \mathrm{~cm}^{-1}$ and the exchangeable or free water $-\mathrm{OH}$ vibrational band at $-3530-3050 \mathrm{~cm}^{-1}$ followed by the ratio of the mineral $\mathrm{OH}$ to the free $\mathrm{OH}$ band.

Table S4. Results from Fourier-transform infrared experiments of montmorillonite (MONT) reacted with glucose a.

\begin{tabular}{|c|c|c|c|c|c|c|c|c|c|c|c|c|c|c|c|c|c|c|c|c|c|c|c|c|c|c|c|c|c|c|c|c|c|}
\hline \multirow[b]{2}{*}{ amount adsorbed } & \multicolumn{3}{|c|}{$\underline{0 \text { minutes }}$} & \multicolumn{3}{|c|}{10 minutes } & \multicolumn{3}{|c|}{20 minutes } & \multicolumn{3}{|c|}{30 minutes } & \multicolumn{3}{|c|}{40 minutes } & \multicolumn{3}{|c|}{50 minutes } & \multicolumn{3}{|c|}{60 minutes } & \multicolumn{3}{|c|}{70 minutes } & \multicolumn{3}{|c|}{80 minutes } & \multicolumn{3}{|c|}{90 minutes } & \multicolumn{3}{|c|}{100 minutes } \\
\hline & $\begin{array}{c}\text { mineral } \\
\mathrm{OH}\end{array}$ & $\begin{array}{c}\text { free } \\
\mathrm{OH}\end{array}$ & ratio & $\begin{array}{c}\text { mineral } \\
\mathrm{OH}\end{array}$ & $\begin{array}{c}\text { free } \\
\mathrm{OH}\end{array}$ & ratio & $\begin{array}{c}\text { mineral } \\
\mathrm{OH}\end{array}$ & $\begin{array}{c}\text { free } \\
\mathrm{OH}\end{array}$ & ratio & $\begin{array}{c}\text { mineral } \\
\mathrm{OH}\end{array}$ & $\begin{array}{c}\text { free } \\
\mathrm{OH}\end{array}$ & ratio & $\begin{array}{c}\text { mineral } \\
\mathrm{OH}\end{array}$ & $\begin{array}{l}\text { free } \\
\mathrm{OH}\end{array}$ & ratio & $\begin{array}{c}\text { mineral } \\
\mathrm{OH}\end{array}$ & $\begin{array}{c}\text { free } \\
\mathrm{OH}\end{array}$ & ratio & $\begin{array}{c}\text { mineral } \\
\mathrm{OH}\end{array}$ & $\begin{array}{l}\text { free } \\
\mathrm{OH}\end{array}$ & ratio & $\begin{array}{c}\text { mineral } \\
\mathrm{OH}\end{array}$ & $\begin{array}{c}\text { free } \\
\mathrm{OH}\end{array}$ & ratio & $\begin{array}{c}\text { mineral } \\
\mathrm{OH}\end{array}$ & $\begin{array}{l}\text { free } \\
\mathrm{OH}\end{array}$ & ratio & $\begin{array}{c}\text { mineral } \\
\mathrm{OH}\end{array}$ & $\begin{array}{c}\text { free } \\
\mathrm{OH}\end{array}$ & ratio & $\begin{array}{c}\text { mineral } \\
\mathrm{OH}\end{array}$ & $\begin{array}{c}\text { free } \\
\mathrm{OH}\end{array}$ & ratic \\
\hline 0 pmole $\mathrm{g}^{-1}$ & 0.00 & 0.27 & 0.00 & 0.00 & 0.27 & 0.00 & 0.00 & 0.27 & 0.00 & 0.00 & 0.27 & 0.00 & 0.00 & 0.27 & 0.00 & 0.00 & 0.23 & 0.00 & 0.04 & 0.13 & 3.00 & 0.03 & 0.07 & 2.57 & 0.03 & 0.03 & 1.00 & 0.03 & 0.02 & 0.70 & 0.03 & 0.02 & 0.56 \\
\hline 2.08 mmole g $^{-1}$ & 0.00 & 0.26 & 0.00 & 0.00 & 0.27 & 0.00 & 0.00 & 0.26 & 0.00 & 0.00 & 0.26 & 0.00 & 0.00 & 0.26 & 0.00 & 0.07 & 0.25 & 3.57 & 0.09 & 0.22 & 2.44 & 0.10 & 0.19 & 1.90 & 0.11 & 0.16 & 1.45 & 0.12 & 0.12 & 1.00 & 0.11 & 0.08 & 0.73 \\
\hline 3.13 umole $\mathrm{g}^{-1}$ & 0.00 & 0.26 & 0.00 & 0.00 & 0.26 & 0.00 & 0.00 & 0.25 & 0.00 & 0.00 & 0.24 & 0.00 & 0.06 & 0.12 & 2.07 & 0.07 & 0.17 & 2.53 & 0.08 & 0.13 & 1.60 & 0.09 & 0.11 & 1.17 & 0.07 & 0.04 & 0.63 & 0.07 & 0.04 & 0.54 & 0.07 & 0.04 & 0.53 \\
\hline $3.42 \mu$ mole g ${ }^{-1}$ & 0.00 & 0.26 & 0.00 & 0.00 & 0.26 & 0.00 & 0.00 & 0.25 & 0.00 & 0.00 & 0.25 & 0.00 & 0.05 & 0.18 & 3.60 & 0.06 & 0.15 & 2.48 & 0.07 & 0.13 & 1.80 & 0.07 & 0.05 & 0.73 & 0.07 & 0.05 & 0.71 & 0.07 & 0.05 & 0.71 & 0.07 & 0.05 & 0.67 \\
\hline 3.47 umole $\mathrm{g}^{-1}$ & 0.00 & 0.26 & 0.00 & 0.00 & 0.26 & 0.00 & 0.00 & 0.25 & 0.00 & 0 & 0.25 & 0.00 & 0.00 & 0.24 & 0.00 & 0.07 & 0.24 & 3.43 & 0.07 & 0.19 & 2.71 & 0.12 & 0.13 & 1.11 & 0.06 & 0.05 & 0.76 & 0.04 & 0.03 & 0.65 & 0.04 & 0.02 & 0.58 \\
\hline $16.64 \mu_{\text {mole g }}^{-1}$ & 0.00 & 0.26 & 0.00 & 0.00 & 0.25 & 0.00 & 0.08 & 0.23 & 3.03 & 0.12 & 0.15 & 1.25 & 0.10 & 0.06 & 0.59 & 0.10 & 0.05 & 0.51 & 0.10 & 0.05 & 0.50 & 0.10 & 0.05 & 0.50 & 0.10 & 0.05 & 0.50 & 0.10 & 0.05 & 0.50 & 0.10 & 0.05 & 0.50 \\
\hline $30.86 \mu$ mole g $^{-1}$ & 0.00 & 0.25 & 0.00 & 0.00 & 0.24 & 0.00 & 0.00 & 0.23 & 0.00 & 5 & 0.16 & 3.06 & 0.06 & 0.10 & 1.69 & 0.07 & 0.06 & 0.81 & 0.05 & 0.03 & 0.58 & 0.05 & 0.03 & 0.56 & 0.05 & 0.03 & 0.56 & 0.05 & 0.03 & 0.56 & 0.05 & 0.03 & 0.58 \\
\hline
\end{tabular}

a $y$-axis value (or absorbance) of the attenuated total reflectance Fourier-transform infrared (ATR-FTIR) spectra vibrational bands of interest: the mineral -OH vibrational band at $-3615 \mathrm{~cm}^{-1}$ and the exchangeable or free water $-\mathrm{OH}$ vibrational band at $-3530-3050 \mathrm{~cm}^{-1}$ followed by the ratio of the mineral $\mathrm{OH}$ to the free $\mathrm{OH}$ band. 
Montmorillonite

0 minutes

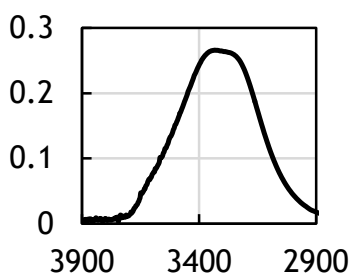

40 minutes
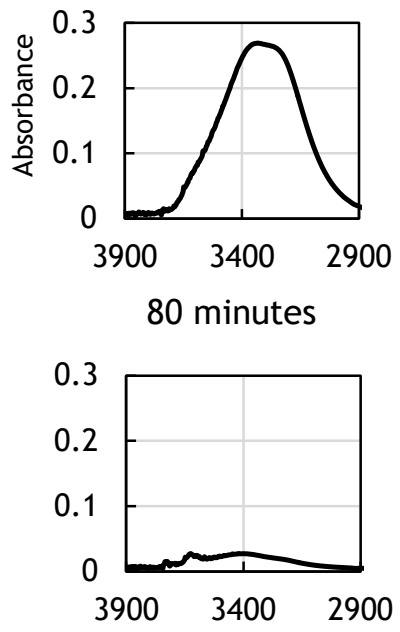

10 minutes

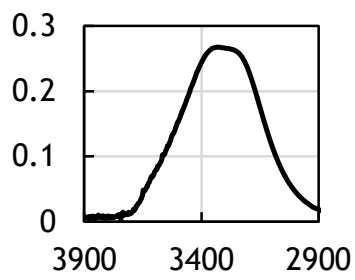

50 minutes
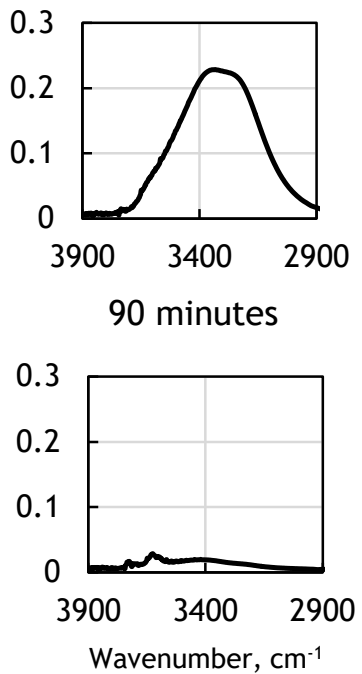

20 minutes

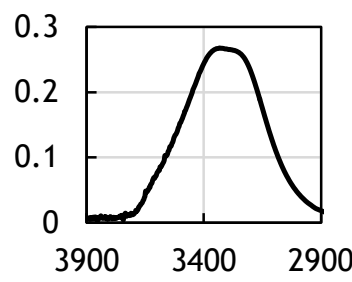

60 minutes

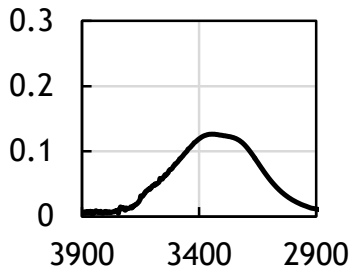

100 minutes

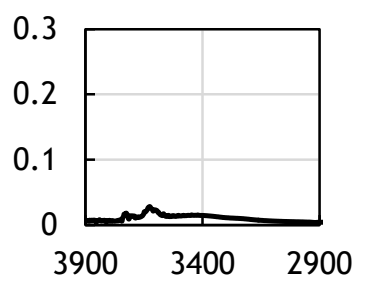

30 minutes
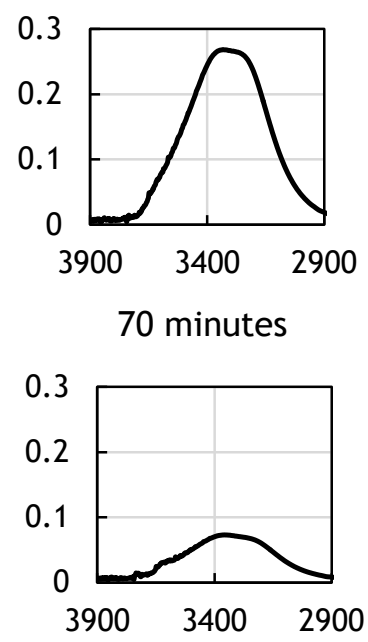

Figure S6. The attenuated total reflectance Fourier-transform infrared (ATR-FTIR) spectra of montmorillonite (MONT) alone over 100 minutes at 10 -min intervals. The exchangeable water population is represented by the broad peak at $3530-3050 \mathrm{~cm}^{-1}$ and mineral $-\mathrm{OH}$ is represented by the sharp peak at $\sim 3615 \mathrm{~cm}^{-1}$. 


\section{$0.66 \mu \mathrm{mol}$ Cello/g clay}

0 minutes

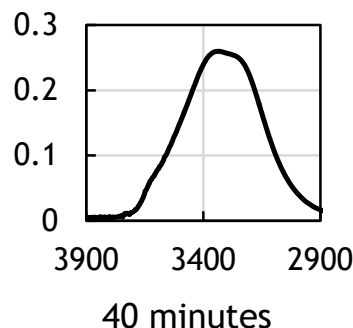

10 minutes

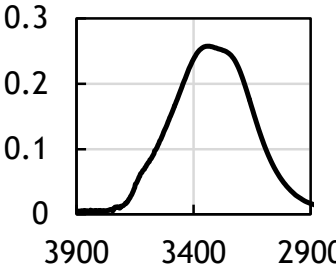

50 minutes

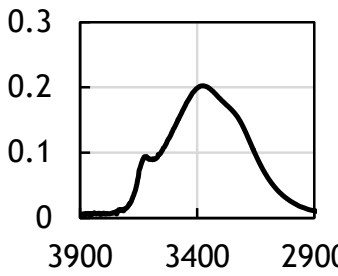

90 minutes

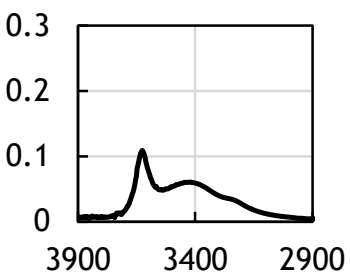

20 minutes

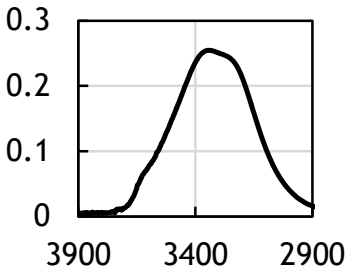

60 minutes

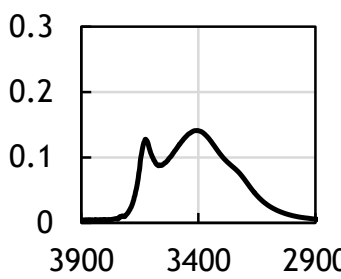

100 minutes

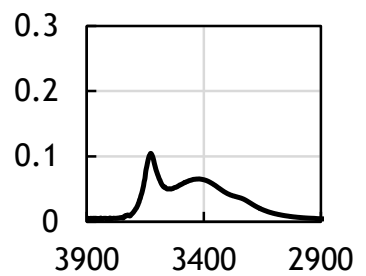

30 minutes

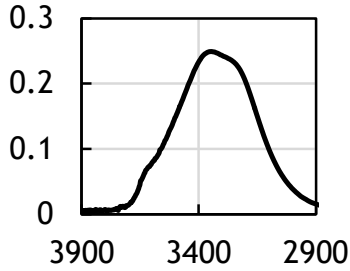

70 minutes

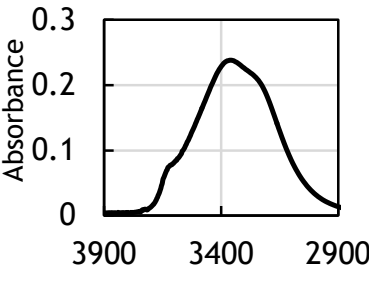

80 minutes

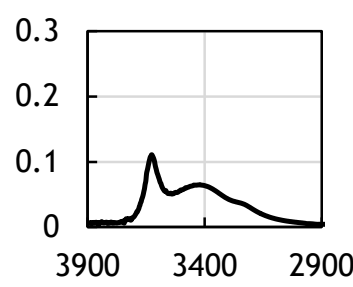

Wavenumber, $\mathrm{cm}^{-1}$

Figure S7. The attenuated total reflectance Fourier-transform infrared (ATR-FTIR) spectra of montmorillonite-cellobiose $(0.66 \mu \mathrm{mol}$ Cello/g clay) over 100 minutes at 10 -min intervals. The exchangeable water population is represented by the broad peak at $3530-3050 \mathrm{~cm}^{-1}$ and mineral $-\mathrm{OH}$ is represented by the sharp peak at $\sim 3615 \mathrm{~cm}^{-1}$. 
0 minutes

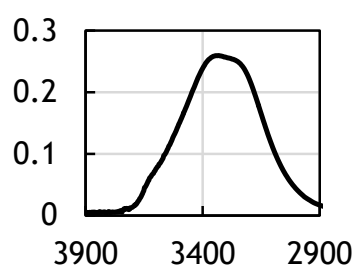

40 minutes

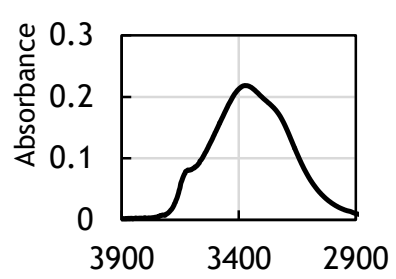

80 minutes

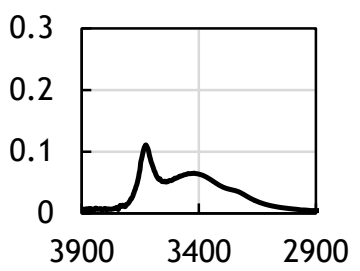

10 minutes

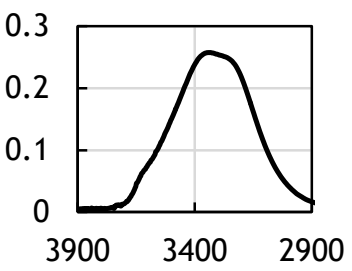

50 minutes

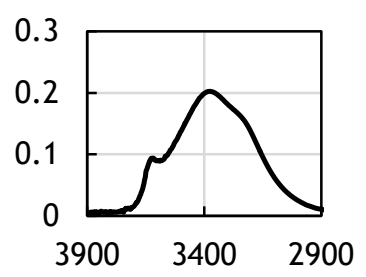

90 minutes

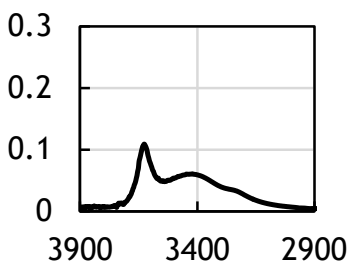

20 minutes

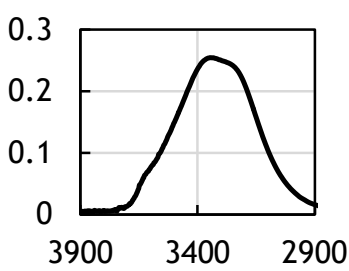

60 minutes

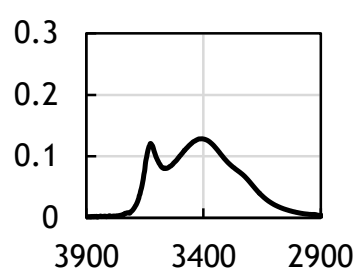

100 minutes

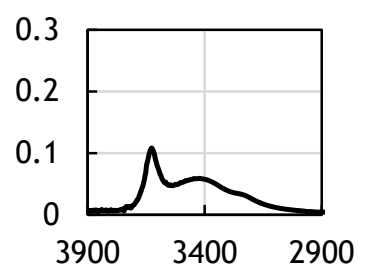

30 minutes

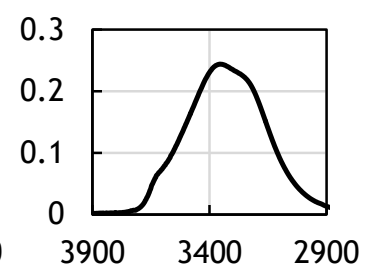

70 minutes

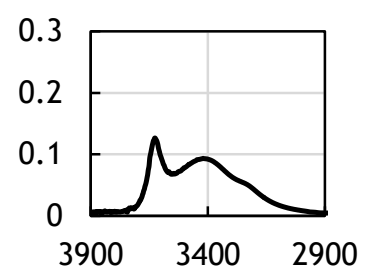

Wavenumber, $\mathrm{cm}^{-1}$

Figure S8. The attenuated total reflectance Fourier-transform infrared (ATR-FTIR) spectra of montmorillonite-cellobiose $(2.19 \mu \mathrm{mol}$ Cello/g clay) over 100 minutes at 10 -min intervals. The exchangeable water population is represented by the broad peak at $3530-3050 \mathrm{~cm}^{-1}$ and mineral $-\mathrm{OH}$ is represented by the sharp peak at $\sim 3615 \mathrm{~cm}^{-1}$. 


\section{$2.64 \mu \mathrm{mol}$ Cello/g clay}

0 minutes
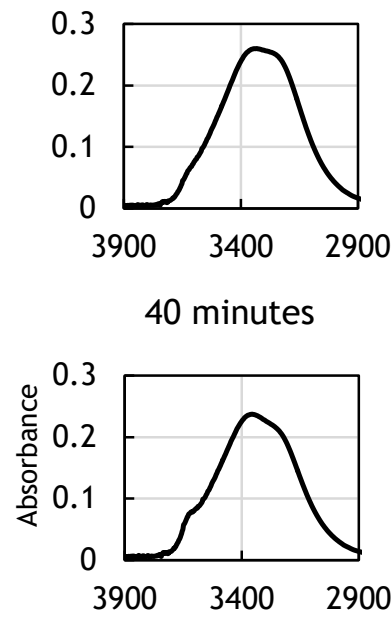

80 minutes

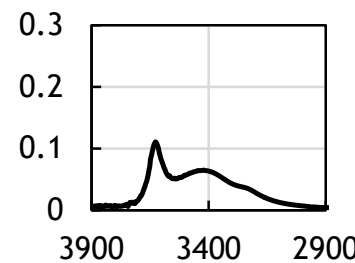

10 minutes
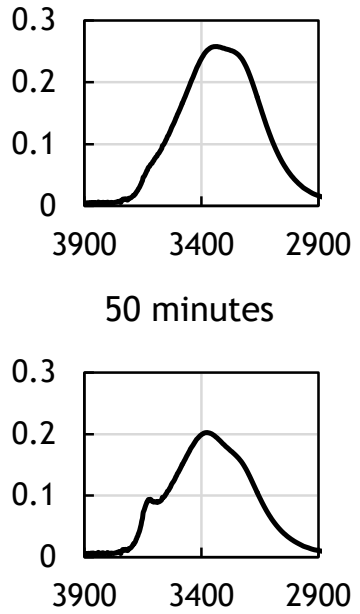

90 minutes

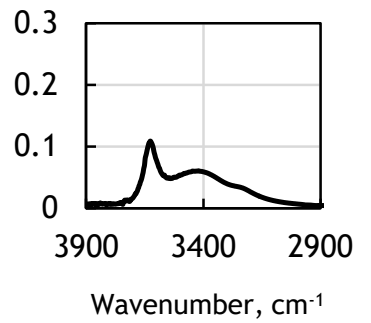

20 minutes
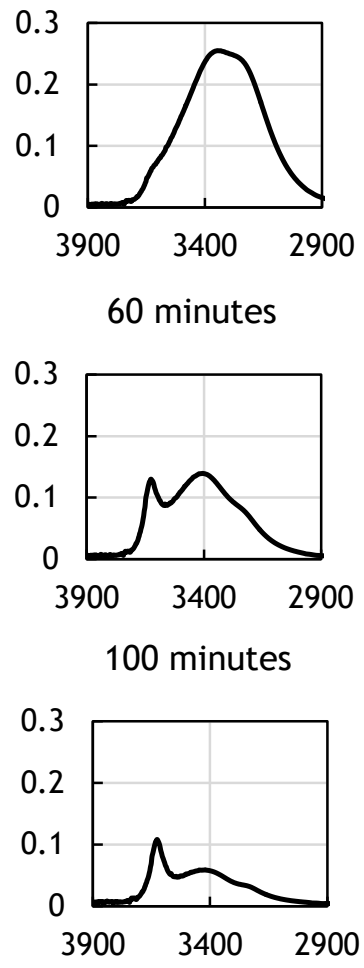

30 minutes
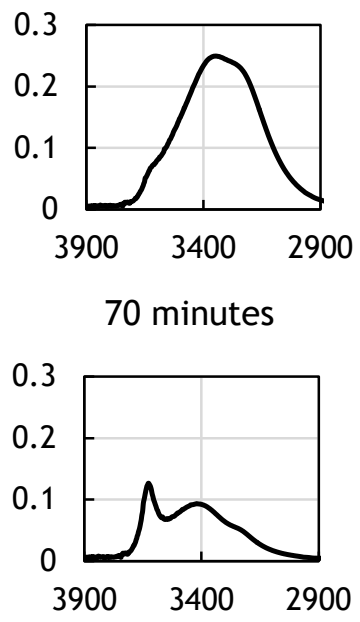

Figure S9. The attenuated total reflectance Fourier-transform infrared (ATR-FTIR) spectra of montmorillonite-cellobiose $(2.64 \mu \mathrm{mol}$ Cello/g clay) over 100 minutes at $10-\mathrm{min}$ intervals. The exchangeable water population is represented by the broad peak at $3530-3050 \mathrm{~cm}^{-1}$ and mineral $-\mathrm{OH}$ is represented by the sharp peak at $\sim 3615 \mathrm{~cm}^{-1}$. 
4.14 $\mu \mathrm{mol}$ Cello/g clay

0 minutes

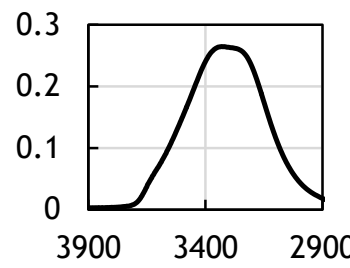

40 minutes

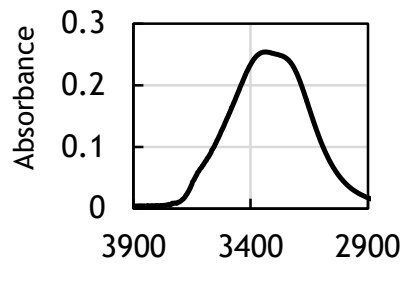

80 minutes

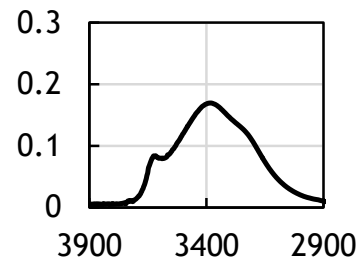

10 minutes

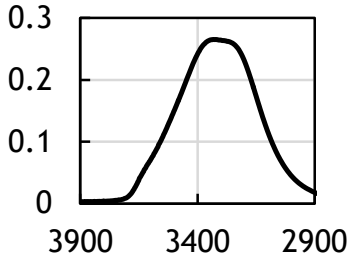

50 minutes
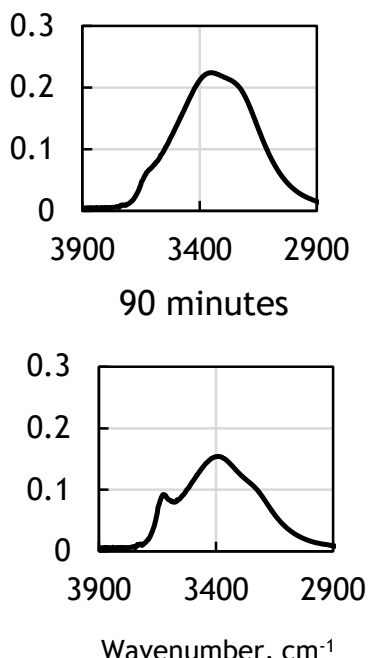

20 minutes

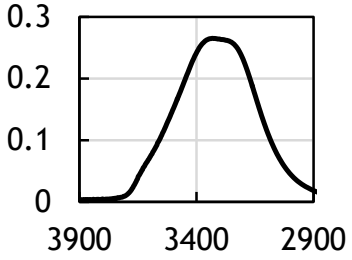

60 minutes

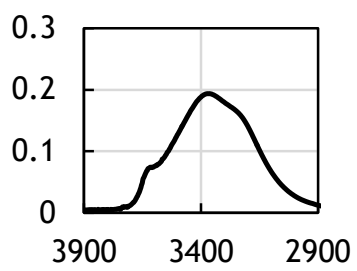

100 minutes

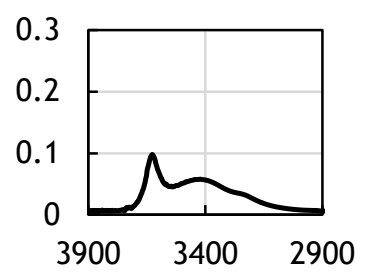

30 minutes

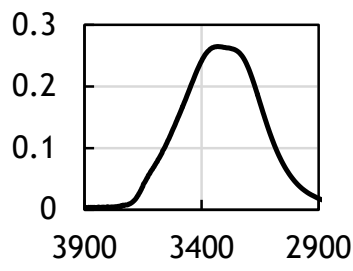

70 minutes

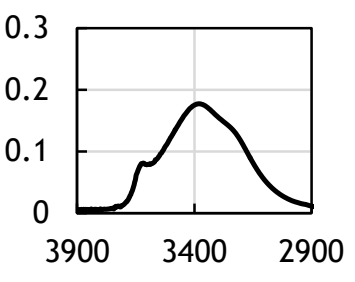


$18.33 \mu \mathrm{mol}$ Cello/g clay

0 minutes

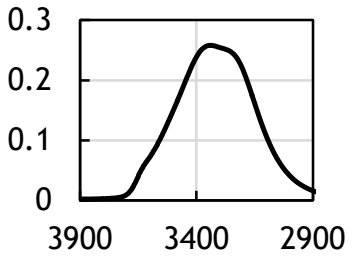

40 minutes
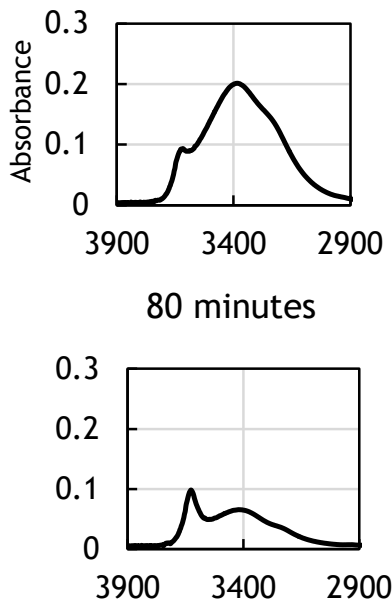

10 minutes

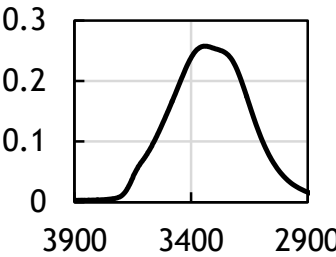

50 minutes
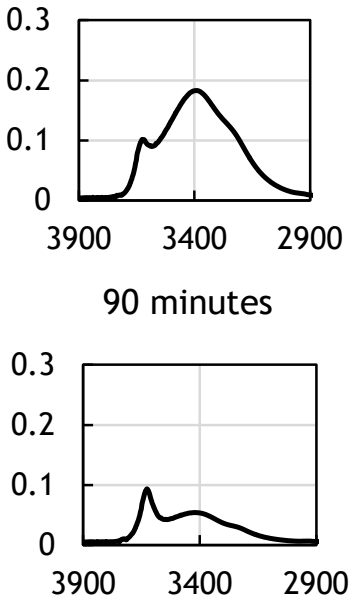

20 minutes

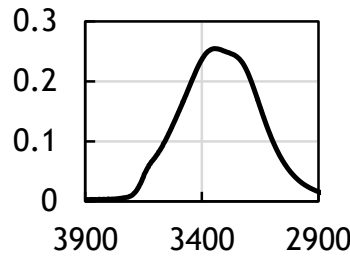

60 minutes
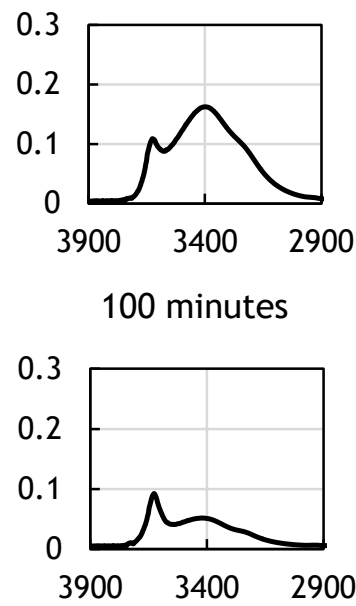

30 minutes

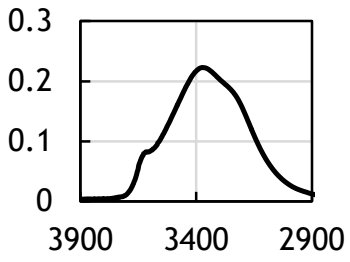

70 minutes

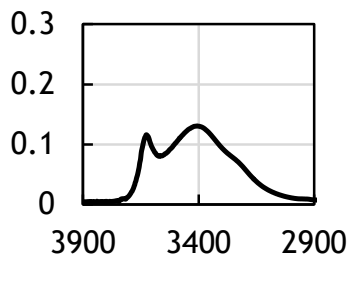

Wavenumber, $\mathrm{cm}^{-1}$

Figure S11. The attenuated total reflectance Fourier-transform infrared (ATR-FTIR) spectra of montmorillonite-cellobiose $(\mathbf{1 8 . 3 3} \mu \mathrm{mol}$ Cello/g clay) over 100 minutes at 10 -min intervals. The exchangeable water population is represented by the broad peak at $3530-3050 \mathrm{~cm}^{-1}$ and mineral $-\mathrm{OH}$ is represented by the sharp peak at $\sim 3615 \mathrm{~cm}^{-1}$. 


\section{$33.23 \mu \mathrm{mol}$ Cello/g clay}

0 minutes

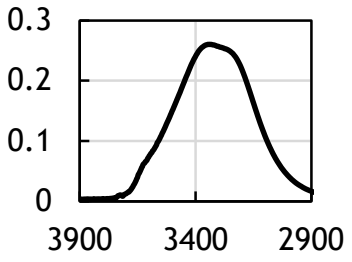

40 minutes

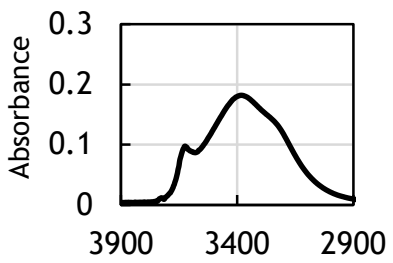

80 minutes

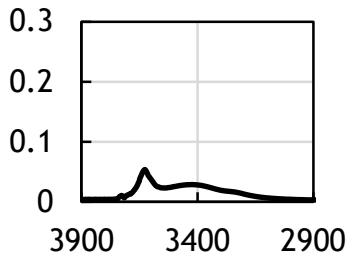

10 minutes

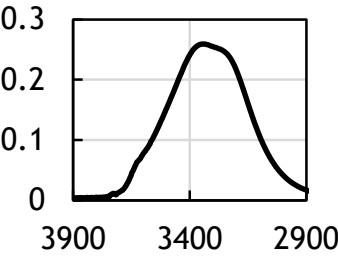

50 minutes

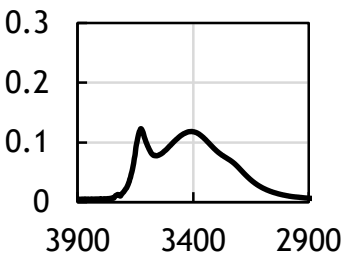

90 minutes

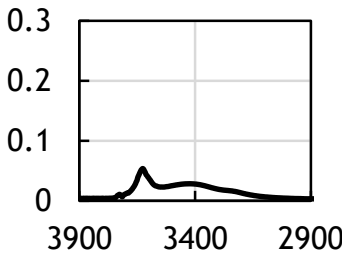

Wavenumber, $\mathrm{cm}^{-1}$
20 minutes

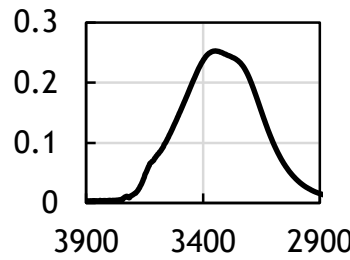

60 minutes

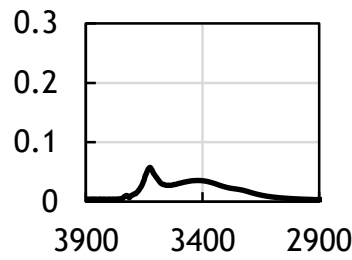

100 minutes

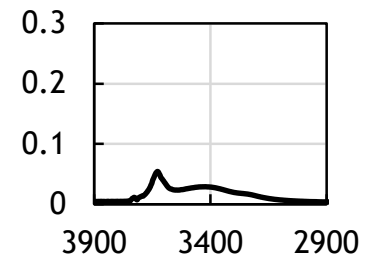

30 minutes

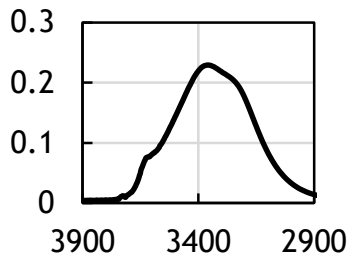

70 minutes

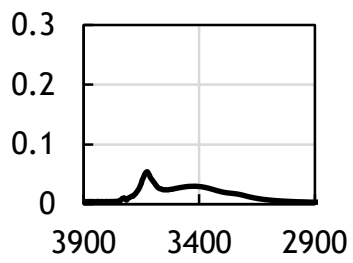

Figure S12. The attenuated total reflectance Fourier-transform infrared (ATR-FTIR) spectra of montmorillonite-cellobiose $(33.23 \mu \mathrm{mol}$ Cello/g clay) over 100 minutes at 10 -min intervals. The exchangeable water population is represented by the broad peak at $3530-3050 \mathrm{~cm}^{-1}$ and mineral $-\mathrm{OH}$ is represented by the sharp peak at $\sim 3615 \mathrm{~cm}^{-1}$. 
$2.08 \mu \mathrm{mol}$ Gluc/g clay

0 minutes

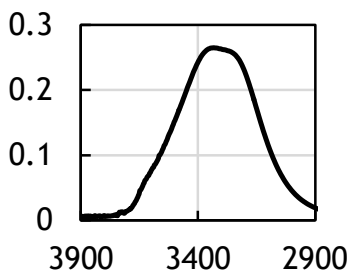

40 minutes
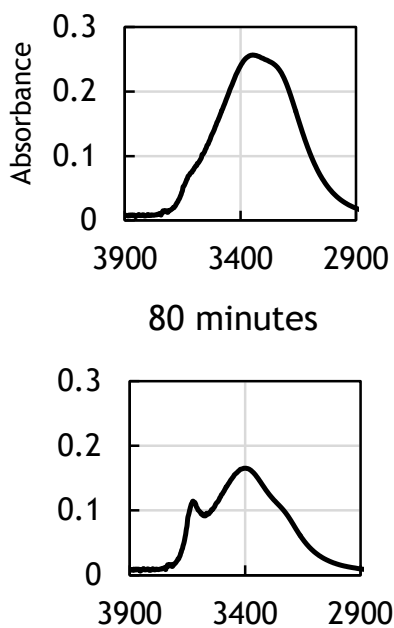

10 minutes
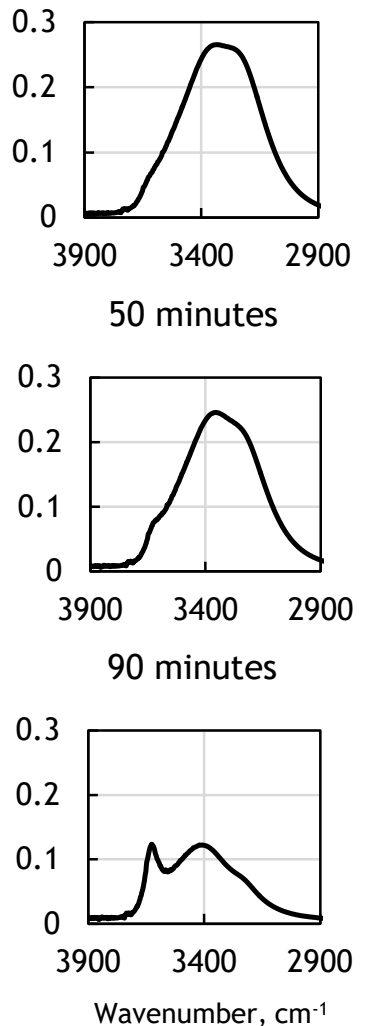

20 minutes
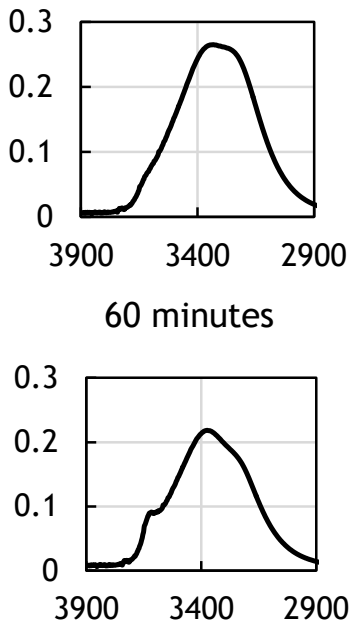

100 minutes

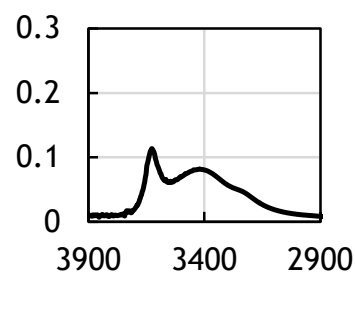

30 minutes
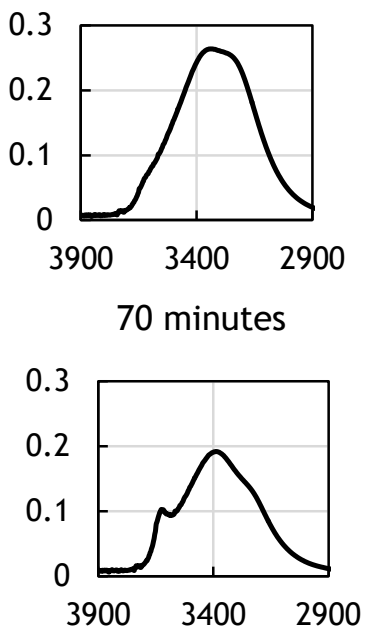
$3.13 \mu \mathrm{mol}$ Gluc/g clay

0 minutes
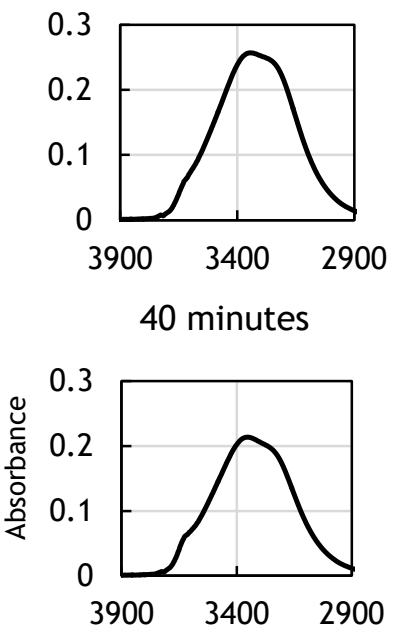

80 minutes

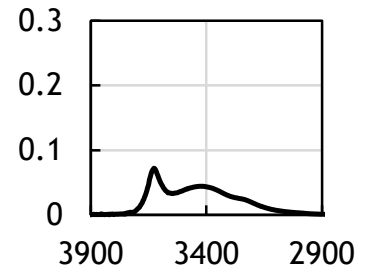

10 minutes
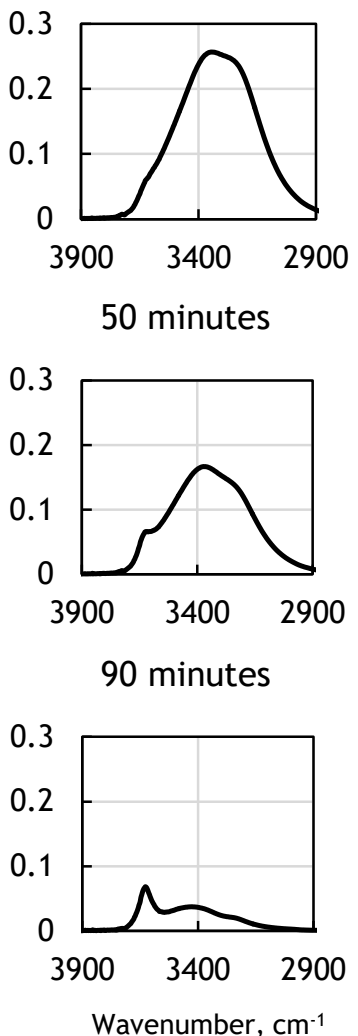

20 minutes
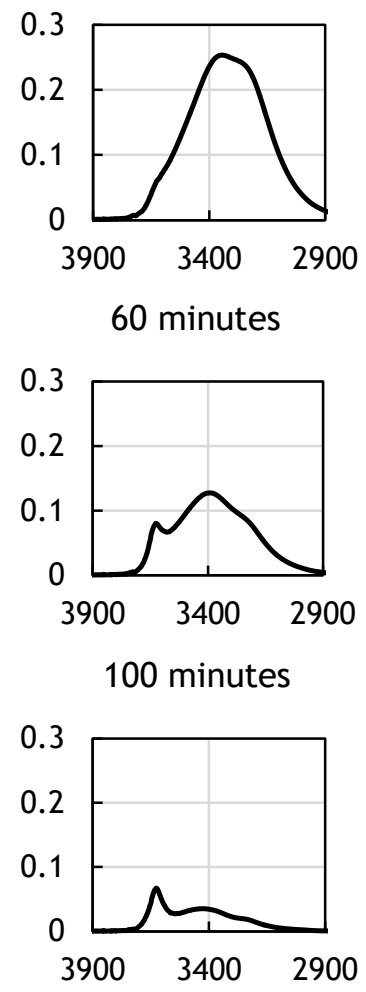

30 minutes
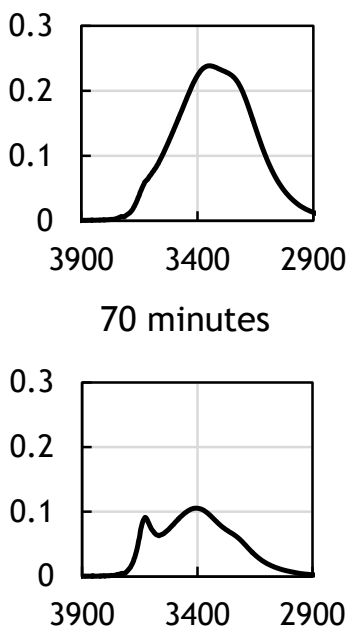

Figure S14. The attenuated total reflectance Fourier-transform infrared (ATR-FTIR) spectra of montmorillonite-glucose $(3.13 \mu \mathrm{mol}$ Gluc/g clay) over 100 minutes at 10 -min intervals. The exchangeable water population is represented by the broad peak at $3530-3050 \mathrm{~cm}^{-1}$ and mineral $-\mathrm{OH}$ is represented by the sharp peak at $\sim 3615 \mathrm{~cm}^{-1}$. 
$3.42 \mu \mathrm{mol}$ Gluc/g clay

0 minutes
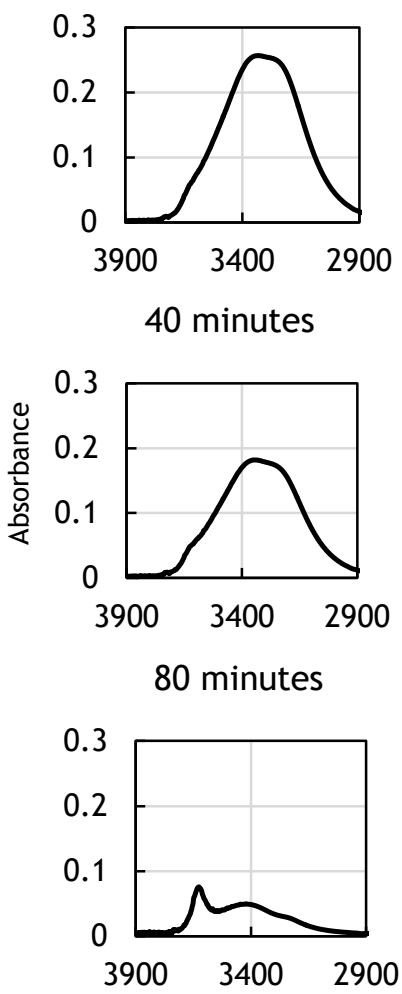

10 minutes
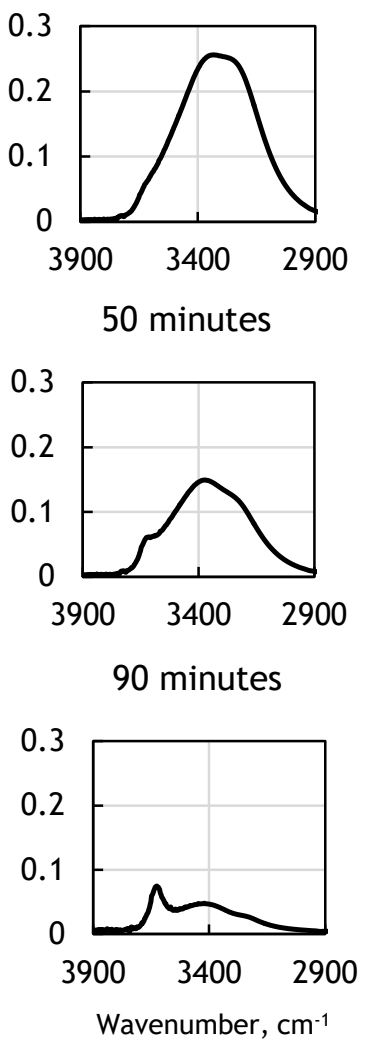

20 minutes
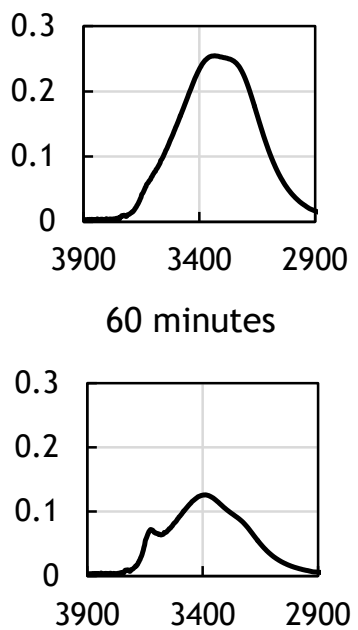

100 minutes

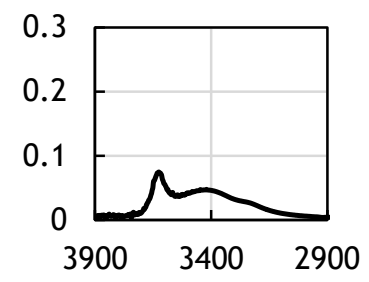

30 minutes
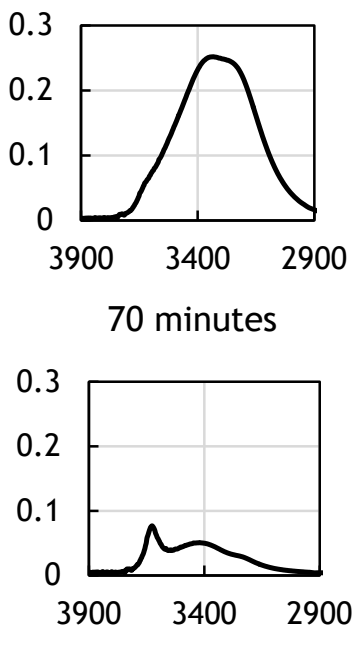

Figure S15. The attenuated total reflectance Fourier-transform infrared (ATR-FTIR) spectra of montmorillonite-glucose $(3.42 \mu \mathrm{mol} \mathrm{Gluc} / \mathrm{g}$ clay) over 100 minutes at 10 -min intervals. The exchangeable water population is represented by the broad peak at $3530-3050 \mathrm{~cm}^{-1}$ and mineral $-\mathrm{OH}$ is represented by the sharp peak at $\sim 3615 \mathrm{~cm}^{-1}$. 
$3.47 \mu \mathrm{mol}$ Gluc/g clay

0 minutes

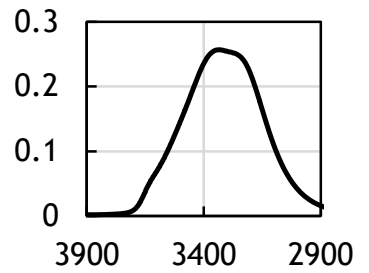

40 minutes

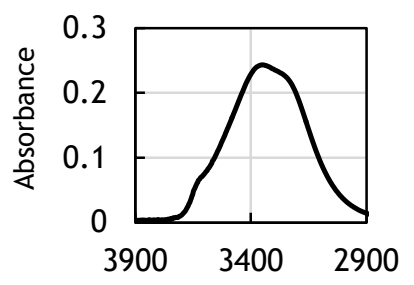

80 minutes

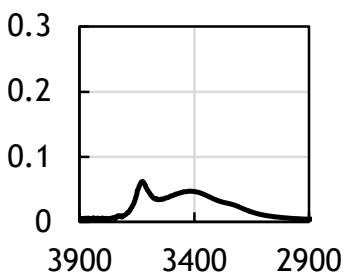

10 minutes

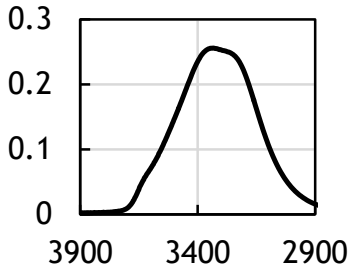

50 minutes
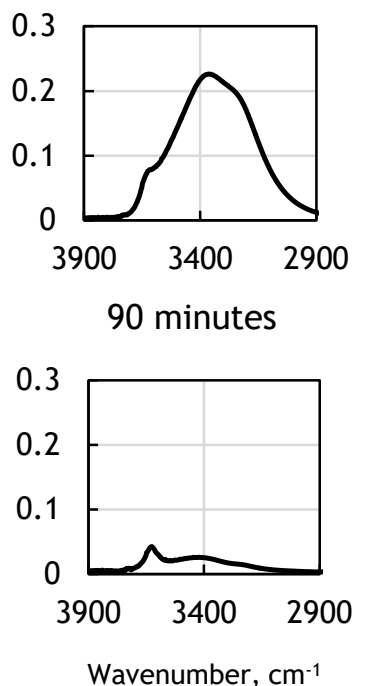

20 minutes

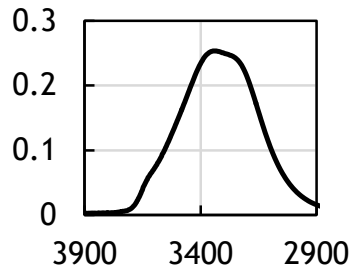

60 minutes

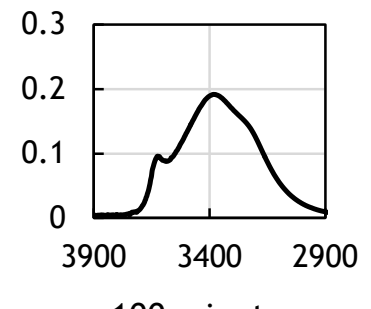

100 minutes

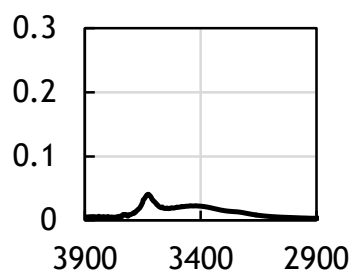

30 minutes
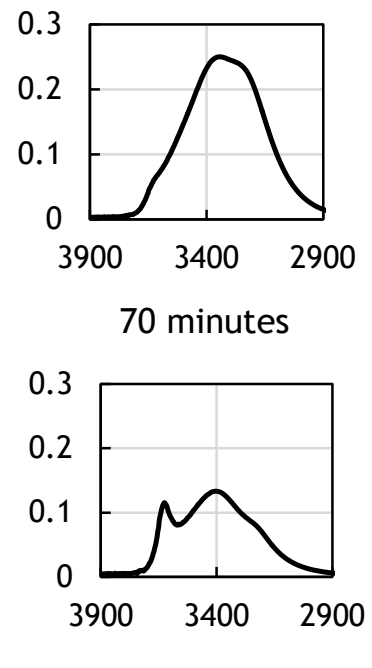

F16. The attenuated total reflectance Fourier-transform infrared (ATR-FTIR) spectra of montmorillonite-glucose $(3.47 \mu \mathrm{mol}$ Gluc/g clay) over 100 minutes at 10 -min intervals. The exchangeable water population is represented by the broad peak at $3530-3050 \mathrm{~cm}^{-1}$ and mineral $-\mathrm{OH}$ is represented by the sharp peak at $\sim 3615 \mathrm{~cm}^{-1}$. 
$16.64 \mu \mathrm{mol}$ Gluc/g clay

0 minutes
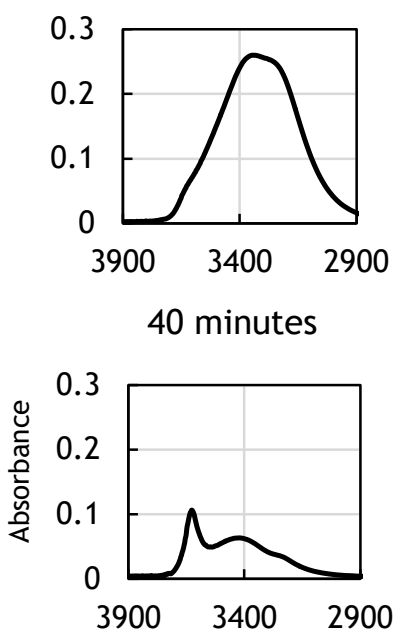

80 minutes

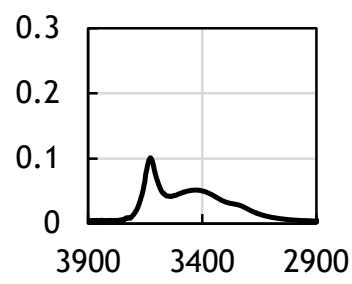

10 minutes
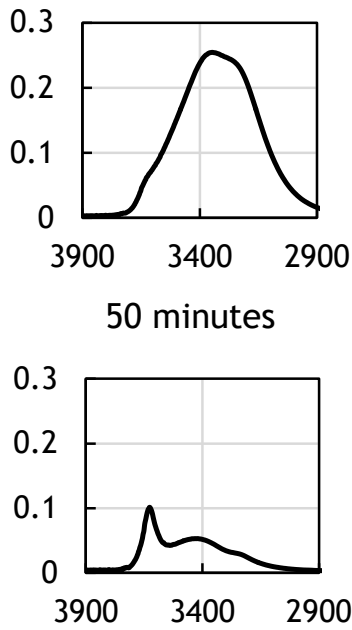

90 minutes

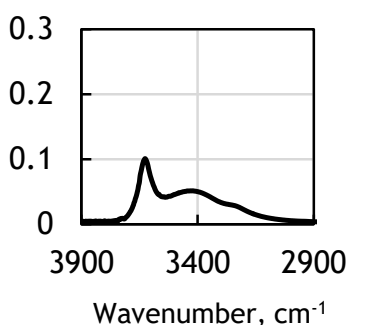

20 minutes
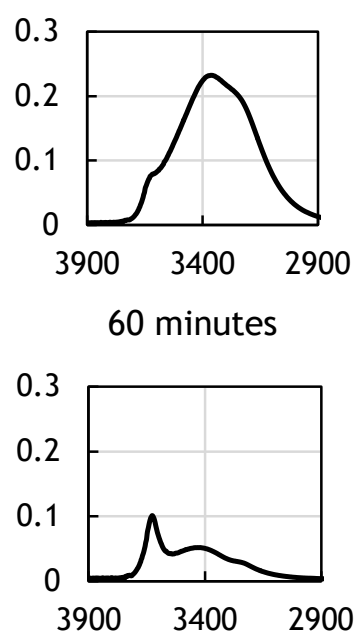

100 minutes

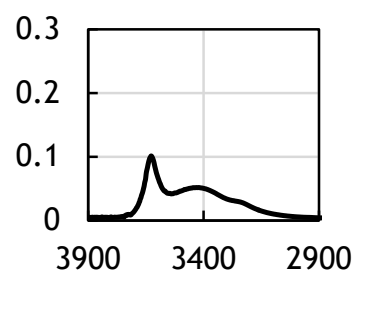

30 minutes
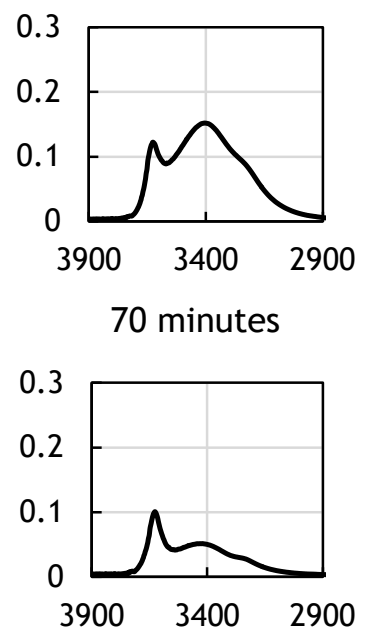
$30.86 \mu \mathrm{mol}$ Gluc/g clay

0 minutes

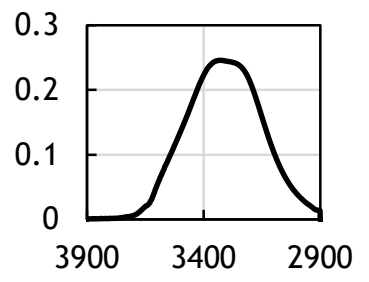

40 minutes

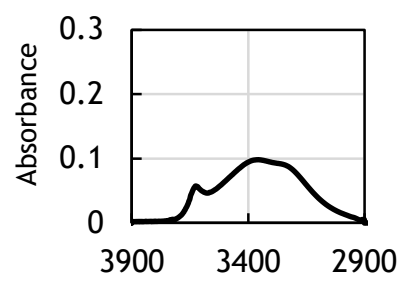

80 minutes

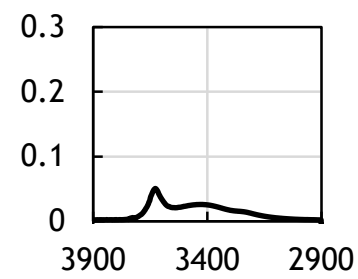

10 minutes
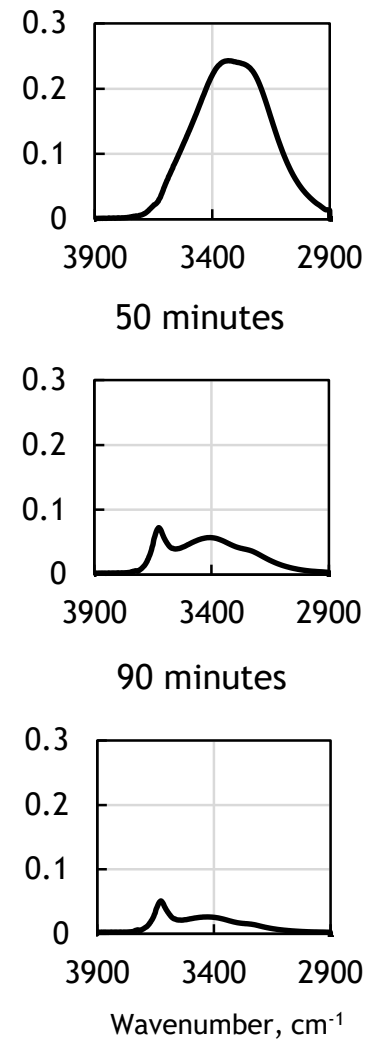

20 minutes
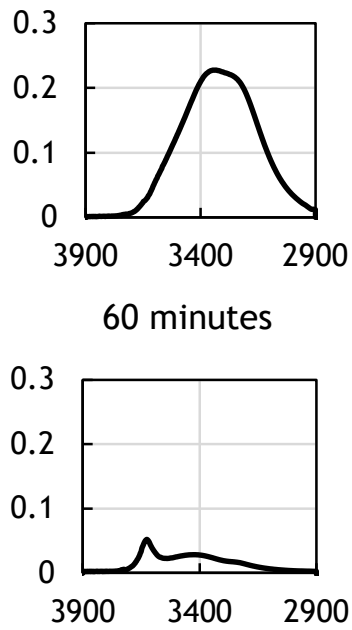

100 minutes

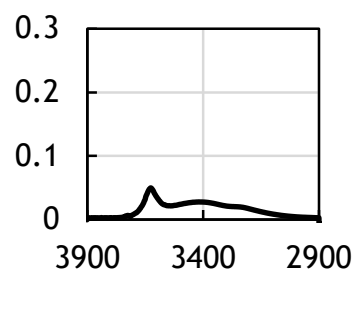

30 minutes
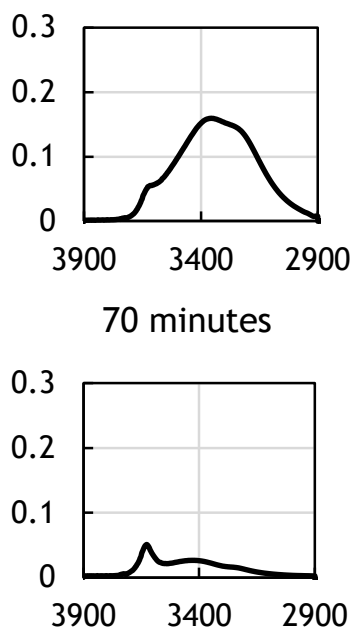

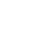

